\title{
Percepción docente sobre la relación maestro-alumno en escuelas secundarias
}

Educational perception on the relation teacher-student in secondary schools

\section{Melanie Itsel Barrios Gaxiola}

\section{Resumen}

Debido a que el trabajo con adolescentes en escuelas secundarias es de suma importancia en educación básica, los maestros deben percatarse de las características y comportamientos que se presentan en la adolescencia para definir las estrategias a implementar durante su enseñanza. Por esta razón, el objetivo es conocer la percepción del docente de secundaria acerca de la relación que existe entre el maestro y el alumno. Tanto el desempeño del maestro como la importancia que el alumno otorgue a la asignatura guiarán a este último hacia la obtención de un aprendizaje significativo. Por medio de estas expectativas, se concluyó que la mayoría de los profesores tratan de mejorar este tipo de relación, sin embargo, hoy en día éste todavía no es el adecuado ya que la etapa de la adolescencia, la gran cantidad de alumnos por grupo y el tiempo son factores negativos que influyen en dicho aspecto. Por lo tanto, se recomienda que el maestro ponga en práctica sus habilidades para mejorar la interacción y convivencia dentro del aula escolar con el propósito de generar en los alumnos motivación e interés por aprender.

\section{Abstract}

Due to working with adolescents in junior high schools is very important in basic education, teachers should be aware of the characteristics and behaviors that are derived from the adolescence stage to define the best strategies to applied in the teaching process. For this reason, the objective in this research is to study the perceptions of high school teachers about the relationship between teacher and student. Therefore, teacher performance and the importance that students have of the subject will lead the latter to obtain meaningful learning. Through these expectations is concluded that most teachers try to enhance this relationship. However, nowadays this is still not adequate because adolescence, the large number of students per group, and time, are negative factors that affect this aspect. Therefore, it is recommended that teachers put into practice their skills to improve the interaction and coexistence in the classroom in order to generate motivation and interest to learn new things.

Palabras Clave: Relación maestro-alumno, práctica docente, adolescencia, motivación.

Keywords: Teacher-student relationship, teaching practice, adolescence, learning motivation. 
Cuando un alumno muestra problemas de aprendizaje de manera generalizada en todas las materias, se pueden establecer algunos supuestos respecto al origen del problema que nos permiten gestionar en un futuro posibles soluciones o, al menos, intervenciones diferenciadas en el proceso educativo (Cervera, 2010). De acuerdo con David Cervera (2010), la inmadurez intelectual es originada por la mala relación maestro-alumno, lo cual también lleva a la pasividad y desmotivación por aprender.

La desmotivación por el estudio, como señala Marchesi (1990), se relaciona con el retraso del alumno en su nivel de aprendizaje, lo cual exige la adaptación a sus metodologías, en la organización escolar o en la oferta educativa, o bien aún en otros recursos para ayudar a los alumnos en su proceso educativo. No obstante, la expectativa que el docente tiene del alumno ha sido motivo de varios estudios a lo largo de los años, entre ellos, destacan los trabajos de Rosenthal y Jacobson (2003) sobre el concepto de "profecía autorrealizable", es decir, las expectativas del profesor son profecías que se cumplen por sí mismas: el alumno tiende a rendir lo que el profesor espera de él (Vieira, 2007).

Es necesario comentar que la relación del alumno con el maestro influye directamente tanto en el rendimiento del alumno como en la percepción social de los compañeros. En esta relación juegan un papel fundamental las variables personales del profesor y del alumno, así como las expectativas del maestro hacia el estudiante, que pueden llegar a modificar la conducta del alumno y las relaciones con sus compañeros. Una buena relación puede garantizar una mejor adaptación al entorno escolar y social (Verdugo, 2000).

Para muchos docentes, la buena relación con sus alumnos es clave para la consecución de los objetivos escolares. La importancia que la buena relación profesor-alumno tiene para un adecuado desarrollo escolar es puesta de relieve en diferentes investigaciones. Cuanto mejor es la relación que se establece entre el docente y el grupo clase, más fácil resulta que éste pueda ejercer su influencia sobre los alumnos. El clima de aula tiene mucho que ver con el tipo de interacciones producidas entre las personas que se desenvuelven en el centro y su entorno (Moreno \& García, 2008). Por lo tanto, con este estudio se pretende ayudar a los docentes a reflexionar y revisar sus concepciones, actitudes y prácticas, además, de transformarlas para que den respuesta a la diversidad de necesidades educativas de su alumnado.

Por otra parte, hablar de las características de los adolescentes de secundaria es muy relevante, ya que el maestro debe considerar los cambios físicos y psicológicos generados en esta etapa. El cambio de humor así como cambio de decisiones repentinas se pueden percibir constantemente en la mayoría de los jóvenes a esta edad, pues en muchas ocasiones la prioridad del adolescente puede ser enfocarse en los estudios, pero, por otro lado, la búsqueda de su propia identidad es inevitable y para lograrlo es común la búsqueda de una relación de amistad con personas que generalmente tienen los mismos intereses.

Tal como Stone y Church (1973) mencionan, el joven trata de independizarse en nuevos aspectos de su vida como en las creencias religiosas o las salidas con personas del sexo opuesto; quiere más privilegios, más permisividad en supervisión y restricciones de los adultos para poder seguir las indicaciones de la pandilla, aunque su sentido de responsabilidad, por las consecuencias de sus propias acciones, sea mínimo. El adolescente mayor comparte las preocupaciones del más joven, pero afronta, además, el problema de su ubicación respecto de todo el mundo adulto, de la independencia y la responsabilidad.

El adolescente, suele defender su postura de anteponer su estética personal delante del grupo a sus necesidades de aprendizaje (Cervera, 2010). Debido a esto, se vuelve doble tarea para el docente impartir los cursos corres- 
pondientes, puesto que debe tomar la iniciativa para establecer las estrategias adecuadas al momento de plantear y planear las actividades. Éstas deben diseñarse conforme a los intereses de los alumnos para lograr en ellos la motivación por el estudio y la superación personal. Asimismo, Piaget (como se citó en Mardomingo, 1994) menciona que el aprendizaje y, por lo tanto, los métodos de enseñanza tienen que adaptarse a las distintas etapas del desarrollo humano.

Por otra parte, durante el periodo de observación y práctica se percibió que los alumnos muestran interés o desinterés por aprender dependiendo del maestro que imparte las clases y no tanto del gusto o agrado por la materia. Por lo tanto, se consideró indispensable tomar en cuenta los comentarios de los docentes en cuanto a su trabajo dentro del aula para conocer cómo es la relación que existe entre el maestro y el alumno. No obstante, hoy en día cada vez se insiste más en el hecho de que las expectativas del profesor acerca del alumno van a influir en la conducta de éste, en su motivación y en definitiva, en el éxito académico. En una serie de estudios realizada por Good y Brophy (1970), se concluyó que la actitud que los profesores tienen hacia sus alumnos influye en la forma cómo actúan con ellos. De este modo, la vida en el aula no es igual para todos; unos tienen más contacto con los profesores que otros (Beltrán \& Bueno, 1995).

Hansen (1998), para expresar cómo se produce la relación del docente con sus alumnos, se refiere a los conceptos de modo: la forma en que trabaja el profesor, el estilo; la manera cotidiana de interacción entre profesor y estudiante, y tacto: la cualidad de mantenerse en sintonía con los estudiantes, captar las situaciones, ser capaz de improvisar y saber aprovechar las oportunidades de comunicación con los alumnos. El autor señala que estas relaciones son más decisivas en la influencia educativa del profesor hacia los estudiantes que los propios contenidos curriculares.
Si bien es necesaria la interacción en el proceso de enseñanza-aprendizaje, también es preciso dar respuesta a las siguientes cuestiones en las cuales se basa la presente investigación: ¿Cuáles son las formas de trabajo del profesor? ¿Qué tipo de comunicación existe entre el maestro y alumno? ¿Se considera al alumno sólo como estudiante o también como adolescente que está pasando por una etapa de cambios? ¿Cómo involucra el maestro las actividades en el aula? ¿Qué se debe enseñar realmente a los alumnos?

A su vez, se requiere que el maestro tenga las habilidades necesarias para integrar el trabajo del adolescente, captar la atención del grupo así como mantener un control adecuado de la disciplina. De tal modo, con los resultados obtenidos se pretende enfatizar los acontecimientos positivos para fortalecer con ello la relación del maestro con el alumno por medio de las interacciones e integraciones colaborativas entre los estudiantes. Asimismo, es de suma importancia incluir los valores dentro del proceso de enseñanza ya que se están formando futuros ciudadanos para su integración a la sociedad.

Amidon y Hunter (1996), definieron la enseñanza como un proceso de interacción que implica, ante todo, la conversación en clase entre el maestro y los alumnos. Podríamos inferir que en dicho proceso el ser humano obtiene una diversidad de conocimientos. De esta manera, poco a poco, con sus capacidades aprende a resolver problemas por sí mismo, los cuales le ayudarán a desarrollar su madurez intelectual y al mismo tiempo actuar de manera participativa en su aprendizaje.

La práctica docente obliga no solamente a tomar en cuenta lo que hacen los maestros, sus prácticas, sino también a estudiar sus condiciones de trabajo, las relaciones institucionales que encuadran su quehacer... cómo determinadas condiciones escolares constriñen o posibilitan el trabajo docente ... historizar la descripción de las estructuras y 
culturas del trabajo docente para reconstruir tendencias, continuidades y rupturas. (Díaz, 2001, p.56).

En la cuestión académica, la interacción del maestro con el alumno es de gran importancia, debido a su vinculación con el aprendizaje que adquieren los alumnos y con el interés que prestan durante la clase. Si no están motivados, aprenden únicamente por lograr una calificación aprobatoria y no por obtener un aprendizaje significativo con el cual resolver futuras situaciones en diferentes aspectos de su vida cotidiana.

Recordando que la adolescencia es una etapa de grandes cambios tanto físicos como emocionales, al trabajar en secundaria con adolescentes se puede presentar una variación de comportamientos negativos de los cuales muchas veces el maestro no puede llevar un control (Anthony, 2011). Tal situación se debe al contexto en el que se encuentren, al tipo de amistades que se tengan y, principalmente, al apoyo que los padres muestran hacia ellos. Los padres, maestros y adultos responsables, por lo general, fomentan una determinada cantidad de conductas independientes durante los años pre adolescentes. Por ello, es tarea tanto del maestro como de los padres de familia guiar al alumno hacia la motivación por el estudio y a la definición de las metas que pretende lograr en un futuro (Duque, 1998).

De acuerdo con Ruiz (2008), la motivación es una predisposición general que dirige el comportamiento a la obtención de deseos. La base de toda motivación es el deseo. Su conexión con el logro dependerá de la voluntad (intención por hacer algo) y ésta se apoyará en la constancia (fortaleza mental del deseo).

Es fundamental que el profesor estimule y tome en consideración las características individuales de cada alumno; que muestre interés real en las consultas personales acerca de sus inquietudes y propuestas. No obstante, en la relación maestro-alumno, es decisiva que el primero se libere de preconceptos o estereotipos y mantenga expectativas positivas y adecuadas con todos los alumnos, pues sin duda, cada uno de ellos podrá ofrecer uno o más aspectos a esa relación que se quiere positiva y constructiva (Vieira, 2007). El objetivo de esta investigación es conocer la percepción del docente de secundaria acerca de la relación que existe entre el maestro y el alumno.

\section{Método}

La presente investigación es de tipo cualitativo ya que se utilizó como método la entrevista a profundidad. De acuerdo con Marradi, Archenti y Piovani (2011), en las ciencias sociales la entrevista a profundidad se refiere a una forma especial de encuentro: una conversación a la que se recurre con el fin de recolectar determinado tipo de información en el marco de la investigación.

\section{Participantes}

En total, fueron 23 docentes entrevistados de 152 que laboran en el turno matutino en distintas escuelas secundarias de Hermosillo, Sonora, México, con el fin de conocer sus perspectivas acerca del trabajo con adolescentes dentro del aula. La mayoría de ellos eran maestros experimentados, con más de 5 años laborando en esta profesión, por lo tanto, no hubo ningún titubeo o duda al momento de responder a cada uno de los cuestionamientos, por el contrario, se les facilitó brindar sus conocimientos respecto al trabajo diario en la escuela secundaria con base en sus experiencias. Dicha distribución por secundaria puede ser analizada en la siguiente tabla: 
Tabla 1.

Número de maestros entrevistados por institución

\begin{tabular}{lcc}
\hline Nombre de la escuela & No. maestros & $\begin{array}{c}\text { No. maestros } \\
\text { encuestados }\end{array}$ \\
\hline Escuela Secundaria Técnica Estatal \#12 & 50 & 5 \\
Escuela Secundaria Estatal \#24 & 32 & 6 \\
Escuela Secundaria Técnica \#66 & 33 & 6 \\
Escuela Secundaria Técnica \#57 & 37 & 6 \\
Total & 152 & 23
\end{tabular}

Algunos de ellos estaban especializados en materias como inglés, matemáticas, español, ciencias, historia y ética. En las entrevistas no se siguió ningún criterio específico; fueron dirigidas a maestros elegidos al azar.

Tabla 2.

Maestros entrevistados por especialidad

\begin{tabular}{lc}
\hline Especialidad & Maestros entrevistados por especialidad \\
\hline Español & 2 \\
Matemáticas & 3 \\
Inglés & 3 \\
Ciencias & 4 \\
Educación Física & 2 \\
Geografía & 0 \\
Historia & 1 \\
Formación Cívica y Ética & 5 \\
Artes & 2 \\
\hline
\end{tabular}

\section{Instrumento}

Dicha entrevista se elaboró con un grupo de investigadores docentes de la Escuela Normal Superior de Hermosillo. Cuenta con diez preguntas de las cuales únicamente se utilizaron las primeras 6 en esta investigación por estar relacionadas al trabajo del docente con adoles- centes y a la relación que existe entre él y los alumnos. Éstas se presentan a continuación:

1. ¿Qué repercusiones tiene para la enseñanza y el aprendizaje en la escuela secundaria considerar a los alumnos como adolescentes y no sólo como estudiantes? 
2. ¿Qué experiencias resultan significativas desde la perspectiva y la problemática de nuestra escuela secundaria actual?

3. ¿De qué manera el conocimiento que el profesor tiene sobre los adolescentes y de su asignatura contribuye a involucrar a los estudiantes en las actividades de aprendizaje?

4. ¿Qué debemos enseñar a los adolescentes en la escuela secundaria y con qué finalidades?

5. ¿De qué forma debe operar la escuela secundaria para garantizar el aprendizaje de los estudiantes?

6. ¿Qué transformaciones convendría hacer a la escuela secundaria mexicana, tomando en cuenta su propia historia, así como las experiencias obtenidas en otros países?

7. ¿Qué actitudes en los alumnos son consideradas como situaciones de riesgo?

8. ¿Cómo maneja usted algunas situaciones en riesgo que se le presentan? Por ejemplo: adicciones, violencia, abusos de diferente tipo, acoso, fracaso escolar, etc.?

9. ¿Qué situación se les presenta con más frecuencia? ¿Cómo la aborda?

10. ¿La institución cuenta con personal de apoyo para atender las situaciones en riesgo? ¿Quién?

\section{Procedimiento}

En cada una de las instituciones se concertó una cita con el director o directora del plantel con el fin de solicitar permiso para realizar el estudio y explicar el objetivo del mismo. Las entrevistas fueron hechas por Licenciadas en Educación Secundaria de manera individual; se explicó a cada uno el propósito de la investigación y la importancia de responder con base en sus experiencias. Ésta tuvo una duración de 30 minutos aproximadamente.

\section{Análisis de datos}

Primeramente, se realizó la trascripción de las respuestas seguido de una categorización por medio de la técnica de colores. Según Stratus y Corbin (2002), ésta consiste en la asignación de conceptos a un nivel más abstracto, las categorías tienen un poder conceptual puesto que tienen la capacidad de reunir grupos de conceptos o categorías. En el momento de agrupar conceptos, también inicia el proceso de establecer relaciones entre estos sobre un mismo fenómeno. Las semejanzas permiten la identificación de una categoría, el esbozo de sus atributos y la especificación de sus condiciones de aparición. Las diferencias entre los casos elegidos hacen posible la elaboración de los atributos de las categorías, la determinación de sus subvariantes y la delimitación de su alcance.

\section{Resultados}

En la primera cuestión, acerca de las repercusiones que tiene en las escuelas secundarias el considerar a los alumnos como adolescentes y no sólo como estudiantes, la mayor parte de las menciones consideró de suma importancia suponer los cambios de la adolescencia sin dejar a un lado sus intereses y aficiones; esto lo discurren como una parte afectiva dentro del proceso de enseñanza-aprendizaje. A su vez, de acuerdo con las respuestas otorgadas por los docentes, el no hacerlo puede provocar falta de concentración en el alumno e incluso la deserción escolar, pues muchas veces de acuerdo con las situaciones y dificultades de la vida diaria del adolescente les es imposible cumplir al $100 \%$ con sus actividades y obligaciones escolares. Por lo tanto, se puede inferir que el profesorado actualmente está preocupado por los cambios y situaciones que el adolescente conlleva en esta etapa. 
Testimonio 20: "El conocimiento sobre su etapa es de gran apoyo para los alumnos. Es necesario valorarlos y saber comprender ante todo lo que están viviendo para ayudarlos a conducirse por el buen camino."

Respecto a las experiencias que resultan significativas desde la perspectiva y la problemática de la escuela secundaria actual, podemos encontrar que el mayor número de menciones que arrojaron los maestros se refiere a la rebeldía por parte de los alumnos, una característica común en la etapa de adolescencia. Lo anterior se corroboró durante las jornadas de observación, cuando tuvieron lugar comportamientos y actitudes negativas entre los estudiantes, grupos numerosos que dan lugar a la indisciplina. De acuerdo con las respuestas obtenidas la relación maestro-alumno y maestro-tutor no eran las adecuadas debido a la falta de comunicación. A su vez, los maestros propusieron que para evitar estas acciones no era adecuado enseñar con el método tradicional sino planificar una clase atractiva, educando al mismo tiempo para la vida y reforzando ciertos programas de apoyo a las escuelas secundarias.

Testimonio 22: "Atendiendo a la pregunta anterior emm... la problemática de las escuelas secundarias pasa por una época de rebeldía de los estudiantes una época de que ellos en su etapa de que no han logrado la madurez completa creen que no haciendo caso no trayendo detalles significantes como cuestiones de disciplina nos provocan un problema muy grave dentro de la escuela."

Por otra parte, la mayor parte de los entrevistados mencionó que el conocimiento del profesor acerca de los adolescentes y su asignatura contribuye a involucrarlos en las actividades de aprendizaje, al motivarlos por medio de la elaboración de actividades que sean del agrado e interés de los alumnos, ya sea utilizando las nuevas tecnologías, haciendo juegos y dinámicas, utilizando las estrategias correctas para cada actividad, etc. Además, los textos deben estar apegados a la realidad para que se logre obtener en los alumnos un aprendizaje significativo. No obstante, poniéndose al nivel de conocimiento del alumno el docente puede entablar con mayor facilidad una conversación con él para entenderlo mejor y tener una mayor cercanía si existe equidad dentro del salón de clases. Tal como lo mencionan tres maestros, la buena relación maestro-alumno influirá en gran medida en el involucramiento de los alumnos hacia las actividades y la adquisición a su vez de una identidad propia.

Testimonio 2: "Ah porque sabemos que el adolescente está en la etapa, de que quiere, la etapa de muy hiperactiva, que quiere mucha actividad, así que ya como maestro yo busco actividades donde él se desenvuelva, por ejemplo en matemáticas, yo hablo de matemáticas, no solamente se trata de resolver los problemas, pero también se trata de que ellos lo comenten, ellos se expresen, porque al adolescente le gusta expresarse y defender su solución, porque al adolescente le gusta defenderse, ya también por ejemplo en matemáticas ellos mismos realizan exposiciones que ellos mismos preparan, que preparan usando, buscando la información del internet porque a ellos les gusta el internet, que preparan utilizando el Power Point, el Excel, el Word, porque a ellos les gusta usar la computadora, entonces las cosas que a ellos les gusta hacer, buscarlas, adecuarlas, para el trabajo diario de la asignatura".

¿Qué debemos enseñar a los adolescentes en la escuela secundaria y con qué finalidad? De acuerdo con la mayoría de los profesores, además de impartir y transmitir conocimientos, lo primordial es inculcar y promover los valores, la cultura y ciertos principios básicos. Por otra parte, mencionan que es necesario encaminar la enseñanza para la vida, para que los alum- 
nos puedan utilizar esos conocimientos adquiridos de manera positiva para salir adelante a pesar de las dificultades que se les puedan presentar. Además, brindarles apoyo psicológico a los alumnos que lo requieran para desarrollar en ellos las habilidades necesarias para tomar futuras decisiones de manera autónoma.

Testimonio 7: "Pues, primeramente es necesario que les inculquemos los valores, esa es la base. De ahí se deriva todo, pues si no existe en ellos la práctica de los valores es muy difícil transmitir los conocimientos que uno como profesor tiene. Por eso es bueno tener buena relación alumno-maestro porque debido a esa interacción uno le puede decir con confianza a los alumnos los errores que tienen para que no los cometan en un futuro, siempre y cuando se les diga con amor no burlándose de ellos. En nuestra asignatura, por ejemplo, yo que tengo la asignatura de historia, les enseño a que relacionemos más esa confianza que tenemos con las actividades, nos ayuda tanto al profesor como al adolescente con el conocimiento porque yo muchas veces también aprendo de ellos"

Los profesores de acuerdo con sus comentarios dan a conocer la forma en la que debe funcionar la escuela secundaria para garantizar el aprendizaje en los estudiantes. Ésta debe ser operada con base en el trabajo colaborativo, es decir, maestros, tutores, directivos y demás personal deben trabajar en conjunto para poder sacar adelante a los alumnos y que egresen con un buen nivel académico. Incluso el apoyo de los padres tiene un gran peso, pues en casa es donde se adquieren los principales valores y se aprende a dar cumplimiento a las obligaciones que le corresponden a cada quien, en este caso, el cumplir con las actividades escolares y obtener buenas calificaciones. No obstante, los maestros mencionan la importancia de estar bien preparados y cumplir con los planes y programas de estudio para que las escuelas estén en constante actualización. Además, el considerar las necesidades y gustos de los alumnos ayudará a garantizar el aprendizaje, una forma de hacerlo es utilizando las nuevas tecnologías de manera formativa para motivar a los alumnos:

Testimonio 17: “¿De qué forma debe operar la escuela? Pues yo creo, como todos estamos en el mismo barco, todos debemos de apoyarnos tanto los directivos como el personal de apoyo, la trabajadora social, el psicólogo, todos estamos en el mismo proyecto y pues todos debemos estar junto con los padres de familia, si el padre de familia no nos apoya nos hace falta, es la parte más importante."

¿Qué transformaciones convendría hacer a la escuela secundaria mexicana, tomando en cuenta su propia historia, así como las experiencias obtenidas en otros países? La mayor parte de las menciones de los maestros considera la posibilidad de invertir más presupuesto en infraestructura y mobiliario, ya que muchas veces ellos cuentan con la capacidad y habilidad de hacer las clases de manera interactiva, sin embargo, carecen de los recursos materiales necesarios para realizarlas $\mathrm{y}$, finalmente, el maestro opta por el método de enseñanza tradicional. El inculcar más los valores, actualizar constantemente el plan de estudio y sus enfoques en cada asignatura así como actualizar las escuelas normales con nuevos métodos de enseñanza son algunas de las transformaciones que ellos recomiendan para la escuela secundaria mexicana. Inclusive, el transformar la escuela secundaria mexicana con base en escuelas de otros países sería una posibilidad de elevar la calidad educativa en nuestro país, ya que de acuerdo con el Foro Económico Mundial, México ocupa uno de los últimos lugares: el sitio 118 de 144 países (Castañón, 2013).

Testimonio 13: "La Secretaría de Educación Pública (SEP) es la que debería hacer cambios, por ejemplo, cuando hacen cambio de poderes por ejemplo, 
antes gobernaba el PRI, era un secretario de educación prista, ahora hay un secretario panista, cada sexenio cambia, esto debe ser a nivel nacional, cada vez hay cambio de poder, hacen muchos cambios, con los que no todos estamos conformes, de nada sirve que yo te diga hay que hacer estos cambios, si de todos modos se van a quedar en el viento. Yo qué más quisiera que le dieran a cada escuela, le dieran cada año, le dieran dinero para que cuando el maestro llegue los mesa bancos estén limpios, que el aula este pintada, cómoda. Los alumnos necesitan motivación herramientas como enciclopedia, buen mobiliario, adecuado, más que nada la infraestructura, los recursos materiales".

\section{Discusión}

Tal como se mencionó anteriormente, la relación maestro-alumno ha sufrido alteraciones a lo largo de los tiempos y esta situación ha hecho que cada vez más el profesor sienta la necesidad de buscar estrategias que le posibiliten una mejor relación con sus alumnos y en consecuencia, el establecimiento de ambientes adecuados para facilitar el aprendizaje en el aula (Beltrán \& Bueno, 1995). Asimismo, el profesor debe cuidar y fomentar su relación humana con todos los estudiantes, pues es fundamental que ante un alumno con resultados académicos deficientes, el maestro mantenga siempre un trato afectuoso y cordial. Además, que esta relación profesor-alumno no se limite al aula. En lo posible, esta relación debe trascender a campos de la formación humana y musical que vayan más allá de los contenidos de clase convencional (Misas \& Tobón, 2007).

Por lo tanto, se reconoce la importancia que tiene el tomar en cuenta las percepciones de los maestros de diferentes asignaturas en cuanto a la relación con sus alumnos y la en- señanza de la clase con el fin de ver qué similitudes y contrastes existían entre una opinión y otra, para con ello, reflexionar en las respuestas que proporcionaron al momento de elaborar las planeaciones didácticas.

Por esta razón, conociendo las opiniones y características de algunos docentes del sector básico, se pretende mejorar y evitar cualquier tipo de acontecimientos conflictivos que se puedan presentar en nuestras futuras clases en secundaria debido a la etapa en la que los alumnos se encuentran.

Finalmente, cabe concluir que las formas del trabajo del profesor van encaminadas a dejar plasmado un nuevo conocimiento en el alumno y a desarrollar una buena relación entre ellos. Sin embargo, se observó claramente que la relación maestro-alumno hoy en día en distintas escuelas secundarias de Hermosillo no fue la más pertinente; el tiempo, la etapa de la adolescencia y la cantidad numerosa de alumnos por cada grupo son factores negativos que comúnmente afectan esta relación. No obstante, cabe mencionar que hay excepciones en las cuales la comunicación entre algunos de ellos es estrecha, así como la buena percepción del profesor sobre el alumno, que influye en sus calificaciones y en los alumnos sobresalientes, tal como se indica en los trabajos de Rosenthal y Jacobson (2003) donde se habla del concepto de "profecía autorrealizable".

Por otra parte, al tratar a los alumnos como adolescentes y no sólo como estudiantes, el profesor intenta involucrarlos en las actividades dentro del aula, relacionando éstas con sus intereses y habilidades, pero, sobre todo, tratando de mejorar la relación maestro-alumno al tener siempre en cuenta la necesidad de educarlos para que sobresalgan en diferentes aspectos cotidianos.

Con todo esto, se recomienda a los docentes que se preocupen por conocer las características del grupo asignado para que los alumnos se interesen por las clases y logren dar sus puntos 
de vista, realicen sus trabajos extraescolares, muestren motivación por aprender y siempre fomenten un ambiente de respeto, colaboración y cooperación entre los mismos compañeros. El proponer un ambiente de este tipo, dará como resultado garantizar un mejor aprendizaje y una formación efectiva en el alumno que lo ayudará a desarrollarse en el ámbito académico como en un futuro profesional.
Amidon, E. \& Hunter, E. (1996). Improving teaching: Analyzing verbal interaction in the classroom. New York: Holt. Rinehart and Winston.

Anthony, D. (2011). La adolescencia: una época de oportunidades. New York: Fondo de Naciones Unidas para la Infancia.

Beltrán, J. \& Bueno, J. (1995). Psicología de la educación. Barcelona, España: Boixareu Universitaria.

Best, J. (1982). Cómo Investigar en Educación. Madrid: Prentice Hall.

Castañón, J. P. (2013). Por una educación de calidad en México. Confederación Patronal de la República Mexicana.

Cervera, D. (2010). Tecnología, investigación, innovación y buenas prácticas. Barcelona: Grao.

Díaz, M. G. (2001). Técnica y Tradición: etnografía de la escuela rural mexicana y de su contexto familiar y comunitario. México: Plaza y Valdéz.

Duque, H. (1998). Los ciclos vitales del ser humano. Tomo 1. San Pablo.

Good, T. \& Brophy, J. (1970). Teachers' communications of differential expectations for children's classroom performance: Some behavior data. Journal of educational Psychology. 61(5), 365-374.

Hansen, P. (1998). Educación intercultural e inmigración en la unión europea. La educación intercultural en Europa. Un enfoque curricular. Barcelona: Pomares-corredor.

Marchesi, A. (1990). Desarrollo psicológico y educación: Trastornos psicológicos y necesidades educativas especiales. España: Alianza Psicología.

Mardomingo, M. (1994). Psiquiatría del niño y del adolescente: método, fundamentos y síndromes. Madrid: Días de santos.

Marradi, A. Archenti, N. \& Piovani, J. (2011). Metodología de las ciencias sociales. Buenos Aires, Argentina: Cengage learning.

Misas, M. \& Tobón, A. (2007). Dicta que dicta. Orientaciones pedagógicas y modelos de ejercicios para el desarrollo de la audición musical. Medellin, Colombia: Universidad de Antioquía.

Moreno, J. \& García, R. (2008). El profesorado y la secundaria: ¿Demasiados retos?. Valencia: Nau llibres.

Rosenthal, R. \& Jacobson, L. (2003). Pygmalion in the classroom. New York: Rineheart \& Winston.

Ruíz, M. (2008). ¿Para qué sirve un líder? Lecciones de liderazgo aplicadas a la empresa. España: Díaz de Santos.

Stone, J. \& Church, J. (1973). Niñez y adolescencia. Buenos Aires: Hormé.

Stratus, A. \& Corbin, J. (2002). Bases de la investigación cualitativa. Medellín: Universidad de Antioquía.

Verdugo, J. (2000). Memoría primer foro de análisis de posgrado. México: Universidad de Colima.

Vieira, H. (2007). La comunicación en el aula: Relación profesor-alumno según el análisis transaccional. Madrid, España: Narcea. 